\title{
Establishment and Implementation of Heating State Assessment of High-voltage Disconnecting Switch Based on RBF Neural Network
}

\author{
Haokai Xie ${ }^{1}$ \\ ${ }^{1}$ School of Electrical and Electric Engineering, North China Electric Power University, Beijing, \\ 102206, China
}

Keywords: Disconnecting Switching, Overheat Fault, RBF Neural Network, State Assessment

\begin{abstract}
Currently, it is difficult to detect the overheat faults of high-voltage disconnecting switch and send out early warning signals effectively and immediately. Hence, an early-warning model was built by using radical basis function (RBF) neural network. The model takes three factors into consideration, including the ratio of load current and rated current, pollution grade and ambient temperature. The test result showed that the overall accuracy rate reached $94.44 \%$, and it can detect the overheat defects with $100 \%$.
\end{abstract}

\section{Introduction}

Disconnecting switch is one of the most widely applied high-voltage electrical equipment with the largest usage amount, the performance and quality of which will directly influence the safe operation of grid. In the event of an accident, large-area blackout will be invited ${ }^{[1]}$. According to operation data ${ }^{[2]}$, from 2008 to 2012, there were 2131 times of overheat fault in disconnecting switch, accounting for $32.1 \%$ of the total. Therefore, overheat control and early warning of disconnecting switch serve as a hotspot and urgent issue to ensure its safe reliability.

In the running of disconnecting switch, there are various factors influencing its overheat, such as excessively high environment temperature, increased contact resistance, excessive load, reduced elasticity of springs, contact erosion by arc and disoperation, etc. This paper selected three factors of the load current, pollution grade and ambient temperature that influence the heating of high-voltage disconnecting switch, established an early-warning model for disconnecting switch overheat fault with the Radical Basis Function (RBF) neural network, and verified the validity of the early-warning model through testing the site data samples.

\section{Reasons for Disconnecting Switch Overheat}

Most disconnecting switch faults occur in conductive shield, main contact, knife-edge pressure finger, switch terminal and the joint between clip and wire ${ }^{[3]}$. When load current flows through these parts, heating increase of disconnecting switch may be caused directly or indirectly, thus triggering overheat fault. The factors inducing overheat fault of disconnecting switch can be mainly divided into two categories, heating factor and heat dissipation factor.

Heating Factors. Load current. Under the effect of load current, heating of disconnecting switch will be arisen, especially at the joints with large contact resistance. larger load current will invite more serious heating.

Pollution grade. Higher pollution grade points to more serious corrosion of the disconnecting switch as well as oxidation of contact surface, hence increasing surface resistance and ultimately aggravating heating[4]. 
Contact resistance. Disconnecting switch overheat point is often the place with relatively large contact resistance.

Heat Dissipation Factors. Heat conductivity mainly relies on air flow. So the main factor influencing the heat dissipation of disconnecting switch is temperature difference between equipment temperature and environmental temperature, i.e., it is subjected to the influence of environmental temperature.

Contact resistance of disconnecting switch is usually measured in out-of-factory test, type test, delivery test and prevention test ${ }^{[5]}$, so it is difficult to conduct live line measurement. In regular substation inspection, what needs to be monitored for the ratio of load current, pollution grade and ambient temperature is data information, so this paper chooses them as the major factors that will influence disconnecting switch heating and adopts these features for evaluating the heating state of disconnecting switch.

\section{Rationale of RBF Neural Network}

RBF neural network is one kind of local approximating neural network, the optimization process of which can be regarded as a surface fitting issue in high dimension space. It can approximate to any non-linear function, hence possessing sound generalization ability ${ }^{[6]}$. It has been successfully applied into areas like time sequence analysis, pattern recognition and fault detection ${ }^{[7]}$.

As shown in Fig.1, RBF network is a feed forward network consisted of input layer, hidden layer and output layer. Hidden layer (or radial basis) is the neuron structure of RBF, and output layer is also called linear layer. The net input of RBF neuron is the distance function multiplied by bias, and radial basis function is also used as activation function.

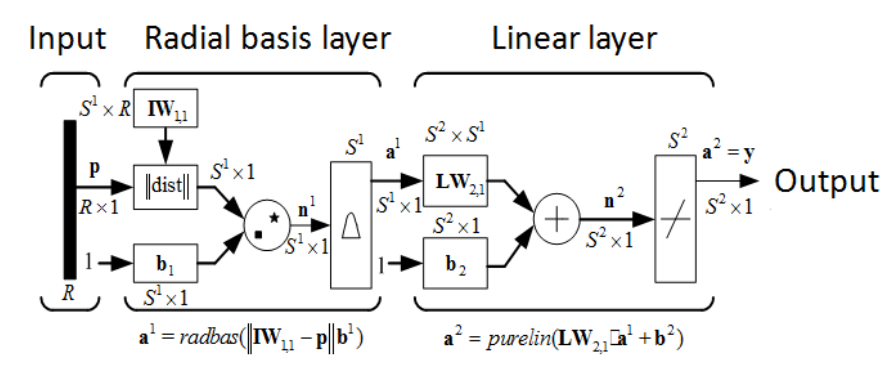

Fig.1 Network Structure of RBF

In the figure, $\mathrm{R}$ refers to input layer and points out input dimension; $S^{1}$ refers to hidden layer formed by radial basis neurons and points out number of neurons; and $S^{2}$ refers to linear output layer. The basic approach of RBF neural network is as follows: RBF as the basis of "hidden" unit is used to form hidden layer space, and maps input vectors onto hidden layer space. When RBF center is determined, the mapping relationship will be set. It turns out direct linear mapping from hidden layer space to output layer space.

This paper adopted the method of self-organizing selection clustering center and took Gaussian function as radial basis function, as shown in formula $(1)^{[8]}$ :

$$
R_{i}\left(\boldsymbol{X}-\boldsymbol{c}_{i}\right)=\exp \left(-\frac{1}{2 \sigma^{2}}\left\|\boldsymbol{X}-\boldsymbol{c}_{i}\right\|\right)
$$

Where, $i=1,2, \ldots, p$, with $\boldsymbol{X}=\left[x_{1}, x_{2}, \ldots, x_{n}\right]$ as the input vector; $c_{i}$ is the $i^{\text {th }}$ center of radial basis function, with dimension being $n$; $\sigma_{i}$ is the Gaussian function variance of $i^{\text {th }}$ neuron in hidden layer; $n$ and $p$ refer to the number of neurons in input layer and hidden layer. 
The output of RBF network is as follows:

$$
y_{j}=\sum_{i=1}^{p} w_{i j} \exp \left(-\frac{1}{2 \sigma_{i}^{2}}\left\|x_{p}-c_{i}\right\|\right)
$$

Where, $j=1,2, \ldots, m$, with $m$ being the number of neurons at output layer; $y_{j}$ is the output value of $j^{\text {th }}$ neuron at output layer; $w_{i j}$ is the link weight value between $i^{\text {th }}$ neuron at hidden layer and $j^{\text {th }}$ neuron at output layer.

Setting up RBF neural network structure requires the network parameter of RBF learning algorithm as the center $\boldsymbol{c}_{i}$ and variance $\sigma_{i}$ of basis function and the weight value from hidden layer to output layer to be $w_{i j}$. The solution steps are as follows ${ }^{[9]}$.

Adopt K-means clustering method to obtain basis function center $c_{i}$.

Solve variance $\sigma_{i}$, and through formula (3) we can get:

$$
\sigma_{i}=\frac{c_{\max }}{\sqrt{2 h}}, \quad i=1,2, \ldots, h
$$

cmax is the biggest distance between the centers selected.

Determine the weight value $w_{i j}$ between hidden layer and output layer. The link weight value among neurons between hidden layer and output layer is obtained through least square method as shown in formula (4):

$$
w_{i j}=\exp \left(\frac{h}{c_{\text {max }}^{2}}\left\|x_{j}-c_{i}\right\|^{2}\right)
$$

Where, $j=1,2, \ldots, m$, and $i=1,2, \ldots, h$.

\section{Model Test}

Early-warning Model. Given that disconnecting switch varies in type and there are many factors that will influence its heating, this paper chose the most widely used GW4-126 series of disconnecting switch with the highest fault rate as target, and adopted three factors of the ratio of load current and rated current, pollution grade and ambient temperature that will influence its heating to evaluate the heating state.

Ratio of load current and rated current. For different types of disconnecting switches, the heating and heat dissipation are related not only with load current but also with rated current. For this, this paper defined the ratio of load current to express the influence of load current strength upon the heating state of high-voltage disconnecting switch, as shown in formula (5).

Ratio of load current=load current/rated current

Pollution grade. This paper quantize the pollution grade of switch according to the regional pollution levels.

Ambient temperature. The paper define the five years' average of highest temperature in fault happens time and place to express the influence of ambient temperature.

Therefore, the input vector $\boldsymbol{X}=\left[x_{1}, x_{2}, x_{3}\right]$ of the RBF neural network established in this paper is as shown in formula (6):

$$
\boldsymbol{X}=\text { [ratio of load current, pollution grade, ambient temperature] }
$$

Hidden layer of RBF neural network adopts Gaussian function in formula (1) as radial basis function; and the output of RBF neural network can provide the specific operation state of high-voltage disconnecting switch, by which whether it is overheating or not can be determined, namely $\boldsymbol{Y}=\left[y_{1}, y_{2}\right]$ : 


\section{Normal state: $\boldsymbol{Y}=[1,0]$ \\ Overheating state: $\boldsymbol{Y}=[0,1]$}

Data Processing and Analysis. To test the validity of the model, this paper chose the influencing factor parameters from 29 groups of operation data under overheating state of disconnecting switch and 27 groups of normal operation data in substations under the control of State Grid Wenzhou Power Supply Company, as shown in Table 1.

Table 1 Data about heating of $110 \mathrm{kV}$ disconnecting switch

\begin{tabular}{ccc}
\hline Year & $\begin{array}{c}\text { Overheat } \\
\text { ing } \\
\text { Data/Gro } \\
\text { up }\end{array}$ & $\begin{array}{c}\text { Normal } \\
\text { Data/Gr } \\
\text { oup }\end{array}$ \\
\hline 2010 & 6 & \\
2011 & 5 & \\
2012 & 5 & 27 \\
2013 & 5 & \\
2014 & 5 & \\
2015 & 3 & \\
\hline
\end{tabular}

From samples in Table 1, 20 overheating samples and 18 normal samples were randomly chosen as the training samples for RBF neural network, with the rest as test samples, including 10 overheating ones and 8 normal ones.

Given that pollution grade is rated from low to high as b, c1, c2, d1, d2 and e1, this paper digitalized it, i.e., $b=1, \mathrm{c} 1=2, \mathrm{~d} 1=3, \mathrm{~d} 2=4$ and $\mathrm{e} 1=5$.

In consideration that the three input features dimensions are different and their sizes also vary, so all samples were normalized as $[0.05,0.95]$ as shown in formula (8) so as to improve the calculation efficiency and convergence speed of neural network and reduce the influence of large input value upon output. To ensure that normalized input features do not run over the range $[0.05,0.95]$ in the test of model, this paper also normalized all ambient temperatures as $-20^{\circ} \mathrm{C}$ to $50^{\circ} \mathrm{C}$.

$$
x^{*}=0.90 \times \frac{x-x_{\min }}{x_{\max }-x_{\min }}+0.05
$$

Set Training speed of RBF neural network being 4 and training error being $1 \mathrm{e}-8$, and then the obtained test results are as shown in Table 2.

Table 2 The heating state test results of $110 \mathrm{kV}$ disconnecting switch

\begin{tabular}{cccc}
\hline State & normal & $\begin{array}{c}\text { Overhe } \\
\text { ating }\end{array}$ & $\begin{array}{c}\text { Recogni } \\
\text { tion } \\
\text { rate } \%\end{array}$ \\
\hline $\begin{array}{c}\text { Normal } \\
\text { Overhe } \\
\text { ating }\end{array}$ & 7 & 1 & 87.5 \\
\hline
\end{tabular}

From Table 2, the early-warning model of overheating state of high-voltage disconnecting switch can recognize all samples in overheating state; even though one normal sample (pink mark) data was mistakenly put under overheating state, i.e., it was distributed in overheating sample (blue mark) area as shown in Fig.2, the categorization accuracy still reached up to 87.5\%. From Fig.4, high ambient temperature and pollution grade tend to invite overheat fault. Meanwhile, in the event of 
large ratio of load current, overheat fault is not for sure; under small ratio of load current, overheat fault will also occur under the condition of high ambient temperature and slow heat dissipation speed. Seen from the overheating samples and normal samples, the overall recognition rate of the model was $100 \times(7+10) / 18=94.44 \%$. Therefore, this model can effectively assess overheat defects for high-voltage disconnecting switch, provide a basis for its state maintenance and cut down blackout accidents caused by overheat defects.

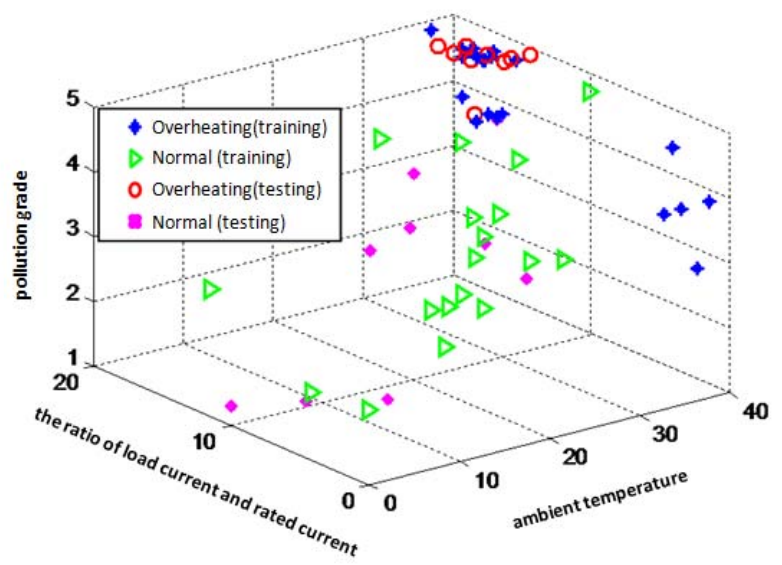

Fig2. Classification results of test samples

Given that the limited number of training samples in this paper, overheat state sample database of high-voltage disconnecting switch should be perfected later to improve the reliability of the early-warning model, which should include samples under various heating conditions, with a view to building a overheat fault early-warning model based on big data. At the same time, the target of this paper is $110 \mathrm{kV}$ disconnecting switch, so later we can build a layered model to divide heating states into various layers, so as to put in place an overheat assessment and early-warning model for the disconnecting switches in different voltage classes.

\section{Conclusions}

In long-term operation, high-voltage disconnecting switch will inevitably be confronted with the problems like mechanical wear, arcing ablation and contact failure, thus leading to overheat.

This paper took into consideration three factors of the ratio of load current, pollution grade and ambient temperature, established an early-warning model for overheat fault of disconnecting switch using RBF neural network, and verified its validity through testing on-site data samples.

This model boasted a $87.5 \%$ recognition rate of samples under normal heating and $94.44 \%$ overall accuracy rate under all heating state evaluation, and realized $100 \%$ early warning of overheat defects.

Against the above three factors influencing the heating of disconnecting switch, maintenance management model was changed, and active maintenance strategy adopted. In addition, this early-warning model was successfully applied to on-site early warning and maintenance.

\section{References}

[1] DL/T 486-2010 《High-voltage alternating-current disconnectors and earthing switches》 [S]. Beijing: China Electric Power Press, 2011.

[2] Yang Kun, Song Gao, Cao Dexin, et al. Experimental study on contact life of disconnecting 
switch[J]. Smart Grid, 2014, 2(2): 63-66.

[3] Liu Xiaobo, Huang Bin, Su Wenyu. Analysis and improved design the overheating problem of the conductive strip of GW4(A)-40.5DW disconnectors[J]. High Voltage Apparatus, 2012, 48(12): 105-108.

[4] Liu Qingsheng, Zhang Lijie, Wang Xintong. Cause analysis of high-voltage disconnecting switch heating and countermeasure[J]. NORTH CHINA ELECTRIC POWER, 2008, 12: 36-38.

[5] Wang Lei, Yan Haojun, Lin Jun, et al. Research on electric contact temperature rise mechanism of outdoor high voltage disconnecting switch[J]. GUANGDONG ELECTRIC POWER, 2014, 27(12): 92-95+117.

[6] Cover T M. Geometrical and statistical properties of systems of linear inequalities with applications in pattern recognition[J]. IEEE Trans. on Electronic Computers, 1965, 3: 326-334.

[7] Chen S. Orthogonal least squares leaning algorithm for radial basis function networks[J]. IEEE Trans. on Neural Networks, 1991, 2(2): 302-309.

[8] He Yaoyao, Xu Qifa, Yang Shanlin, et al. A power load probability density forecasting method dased on RBF

neural network quantile regression[J]. Proceedings of the CSEE, 2013, 33(1): 93-98.

[9] Mu Yunfeng. Study on the application of RBF neural network learning algorithm for pattern classification[D]. Dalian: Dalian University of Technology, 2006. 